\title{
THE GOAL AND MODERN TENDENCIES OF THE LABOR MARKET POLICY
}

\section{SALOME KOBAKHIDZE}

\section{PhD student}

Ivane Javakhishvili Tbilisi State University, Georgia

salome.kobakhidze@tsu.ge

Abstract. Inclusive and well-functioning labor markets are a prerequisite for the economic strength of a country. This includes ensuring enough jobs and social justice protection. Having a job is an important factor for material well-being, security, equality and personal human development, but in Georgia the status of an employee is not associated with normal living conditions. Due to high level of poverty in the country, the population agrees to work for an inadequate fee. The part of the informal sector in the labor market is quite high, this is mainly associated with low pay, unbearable conditions and the absence of safety standards. In such conditions, the main task of the government is to protect the social rights of workers, reduce the informal sector of the labor market, and implement effective and non-discriminatory labor market and employment policies.

\section{KEYWORDS: LABOR MARKET, EMPLOYMENT POLICY, LABOR FORCE, STRUCTURE OF UNEMPLOYMENT, STRUCTURAL} UNEMPLOYMENT, “UNSATISFIED” WORKERS

For citation: Kobakhidze S. (2020). The Goal and Modern Tendencies of the Labor Market Policy. Globalization and Business, №9, pp. 35-41 (In Georgian). https://doi.org/10.35945/gb.2020.09.004 


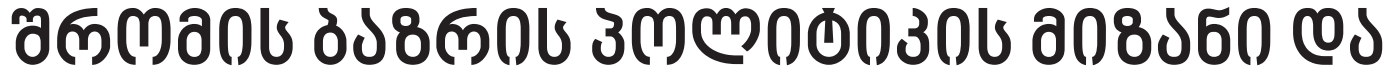

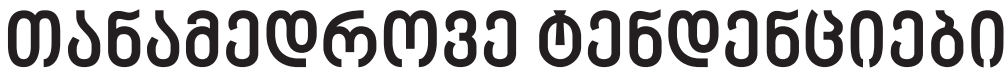

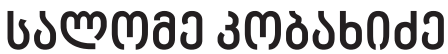

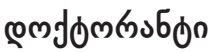

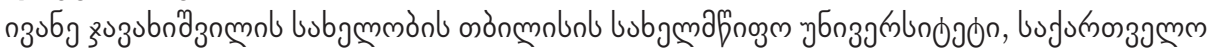

salome.kobakhidze@tsu.ge

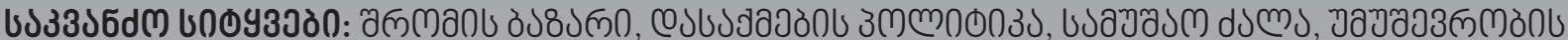

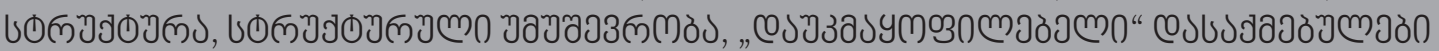

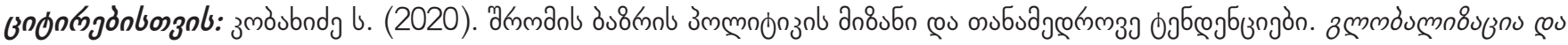
bo86glon, №9, 33. 35-41. https://doi.org/10.35945/gb.2020.09.004

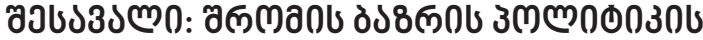

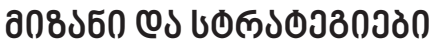

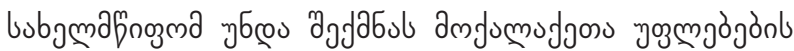

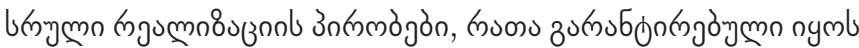

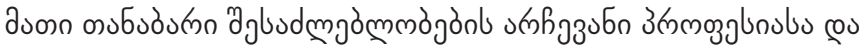

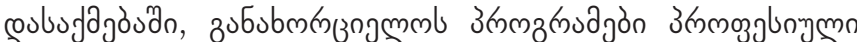

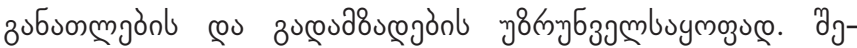

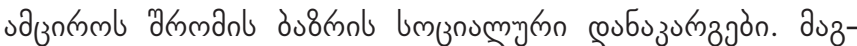

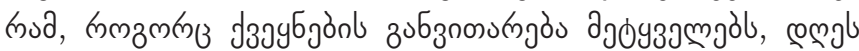

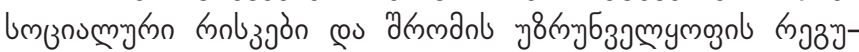

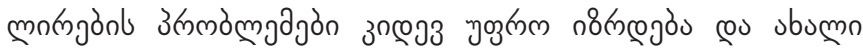

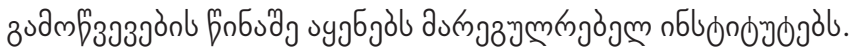

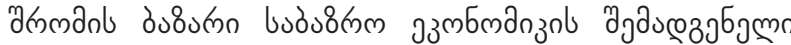

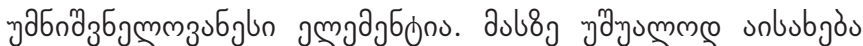

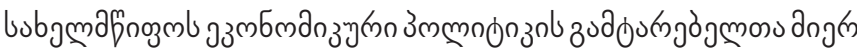

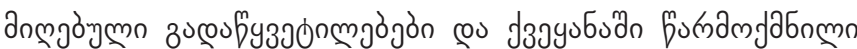

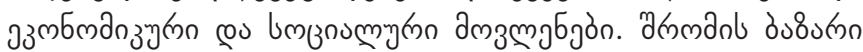

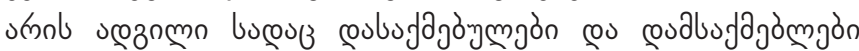

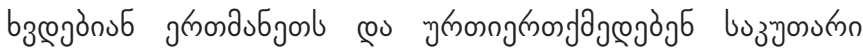

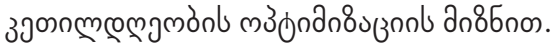

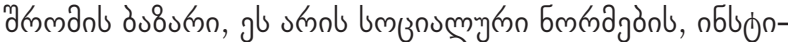

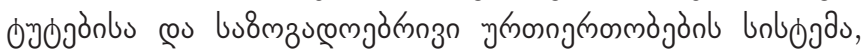

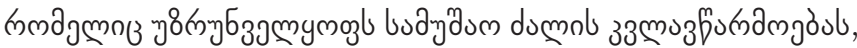

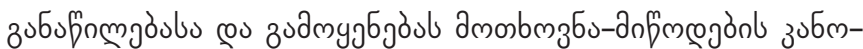

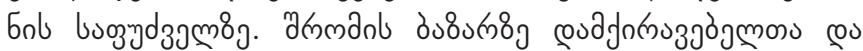

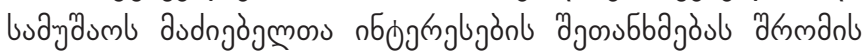

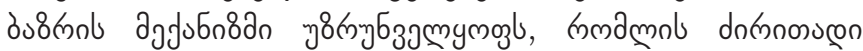

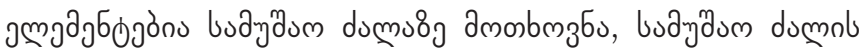

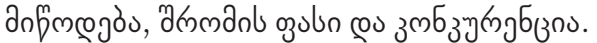

ذзауง

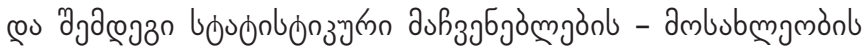

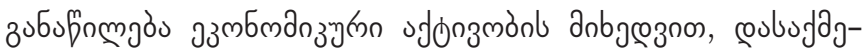

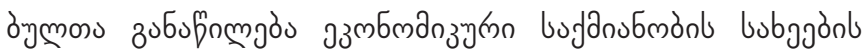

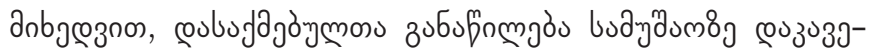

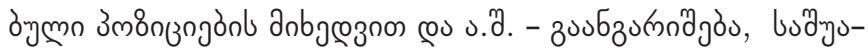

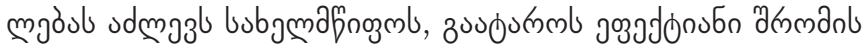

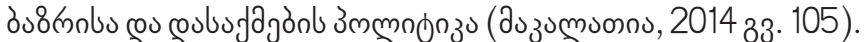

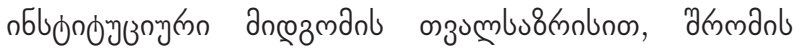

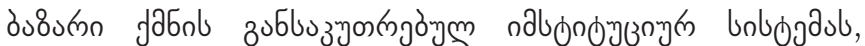

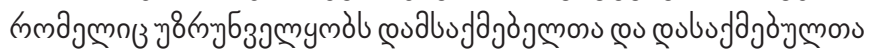

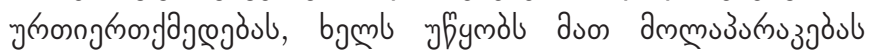

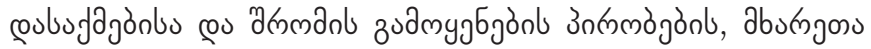

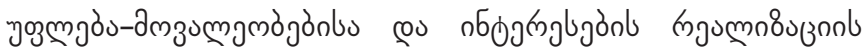

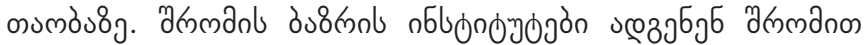

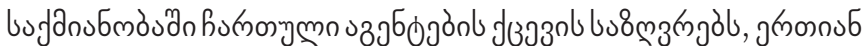

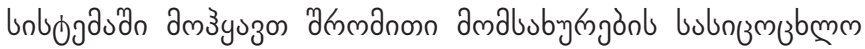

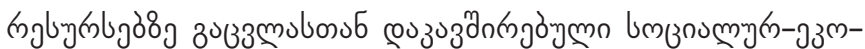

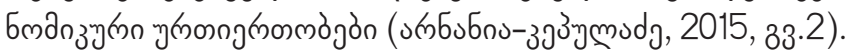

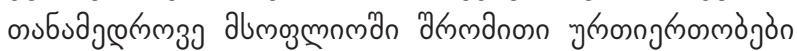

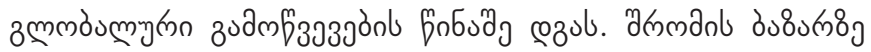

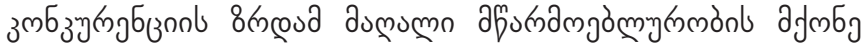

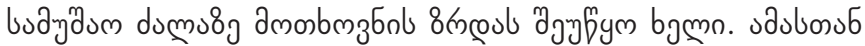

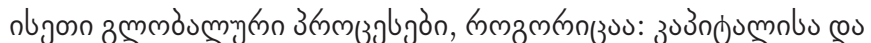

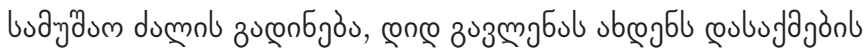

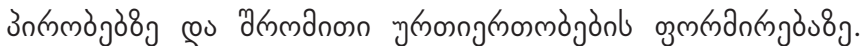

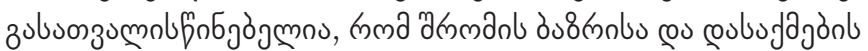

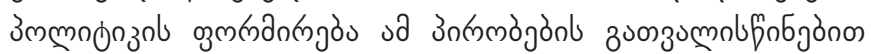

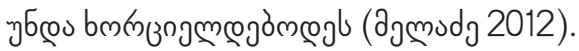

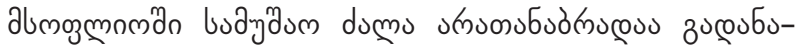

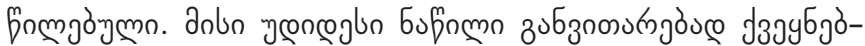

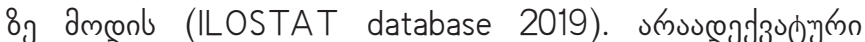




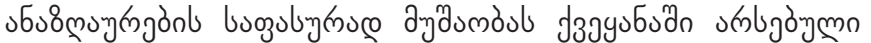

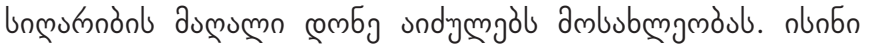

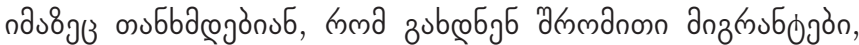

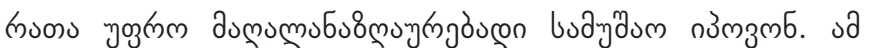

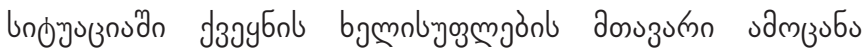

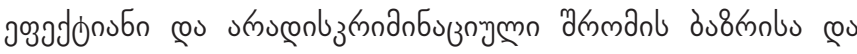

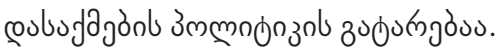

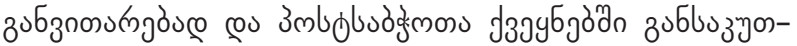

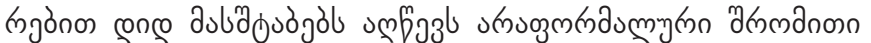

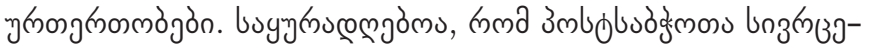

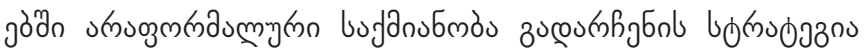

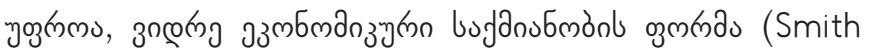
and Stenning, 2006. P. 192, Aliyev, 2015. P.8). Jrmmanl

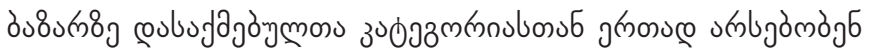

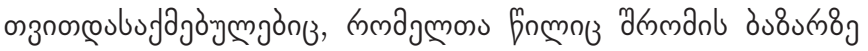

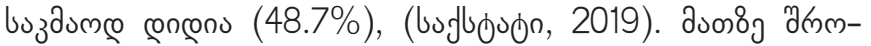

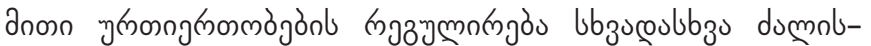

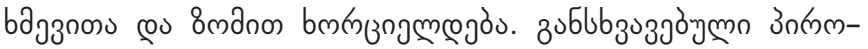

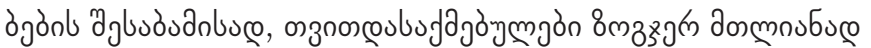

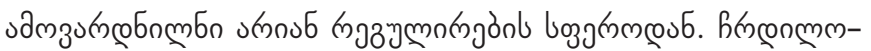

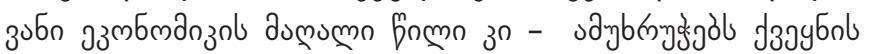

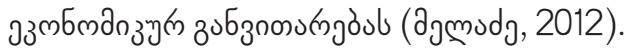

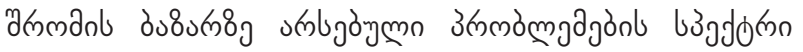

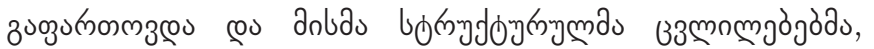

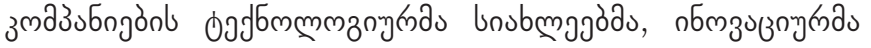

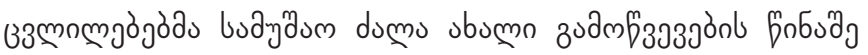

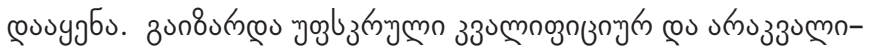

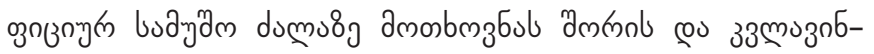

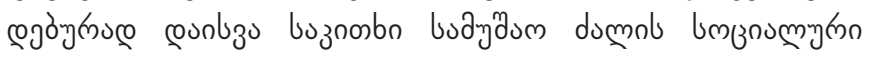

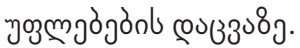

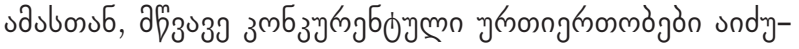

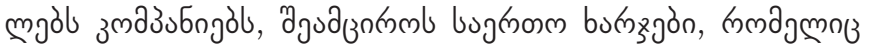

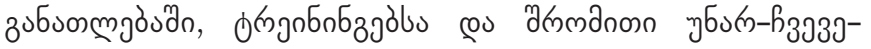

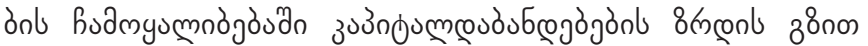
bmingrommegos.

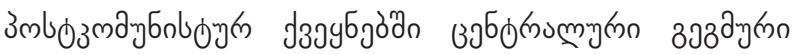

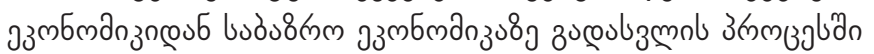

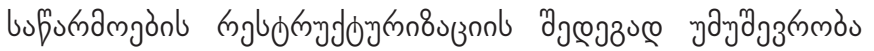

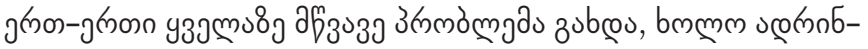

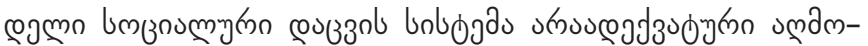

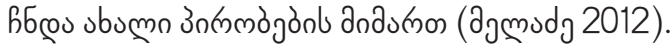

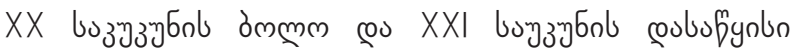

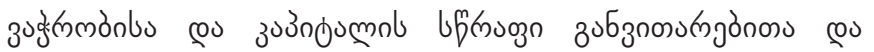

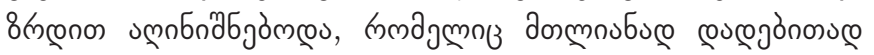

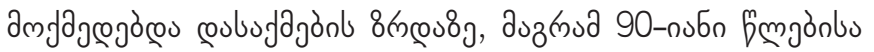

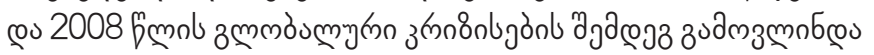

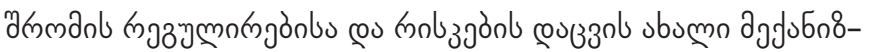
ajon oुm

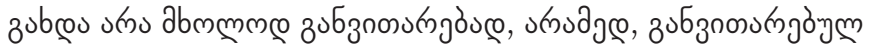

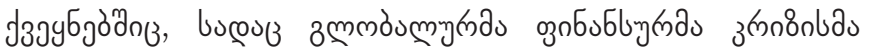

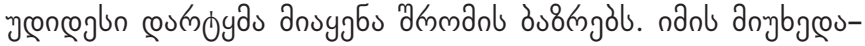

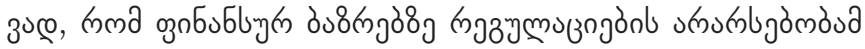

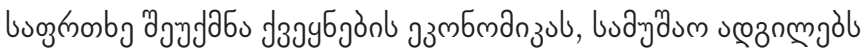

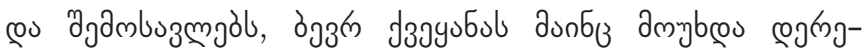

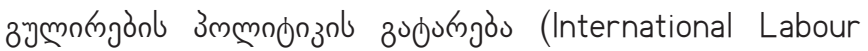
Organization, 2018, p. 3-4, 26).

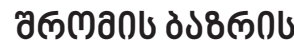

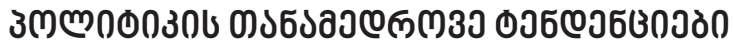

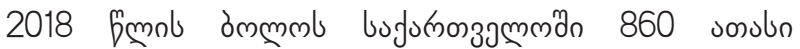

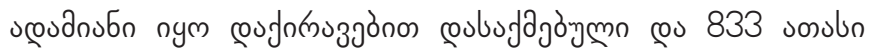

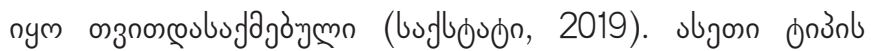

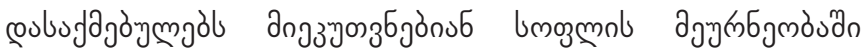

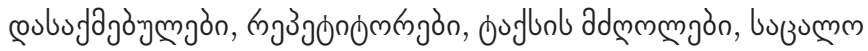

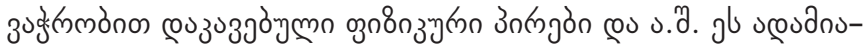

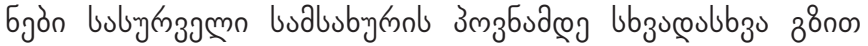

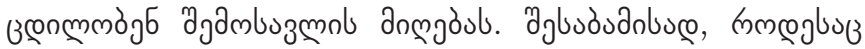

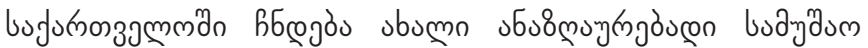

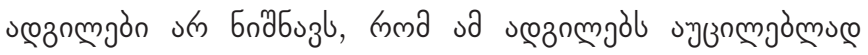

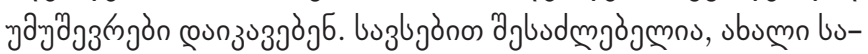

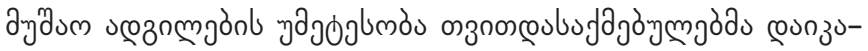

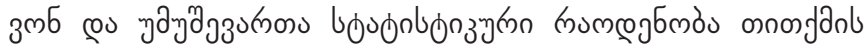

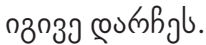

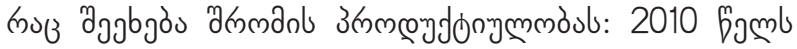

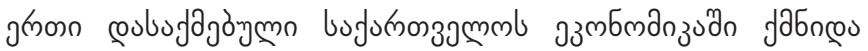

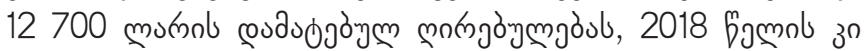

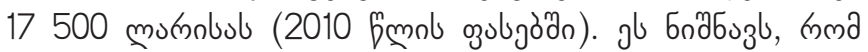

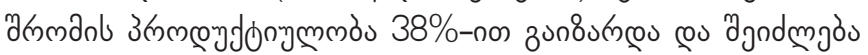

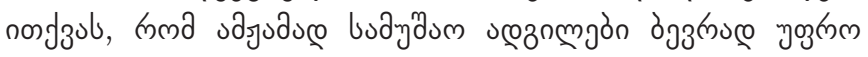

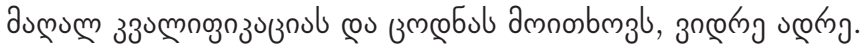
(buflododon 2019, buahuzodg, 2019).

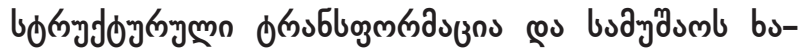

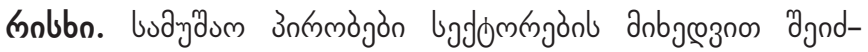

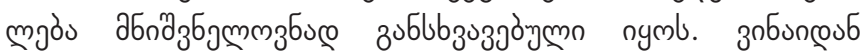

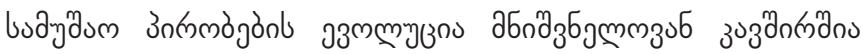

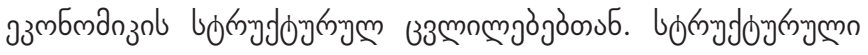

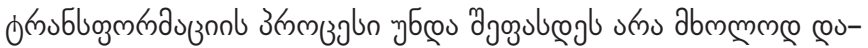

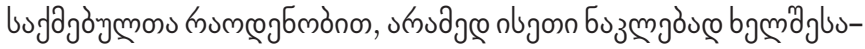

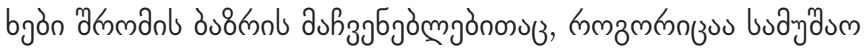

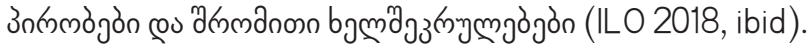

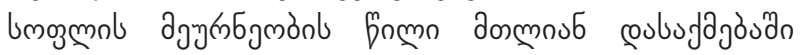

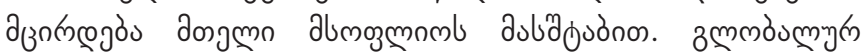

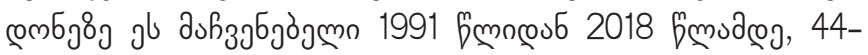

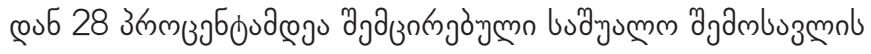

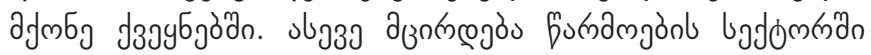

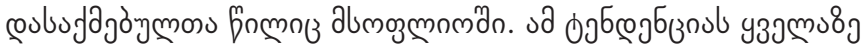

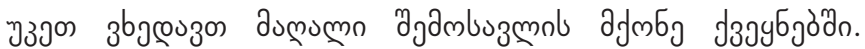

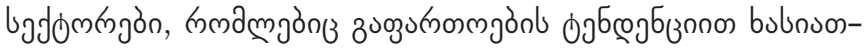

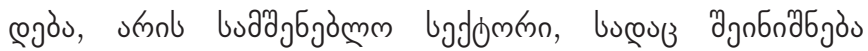




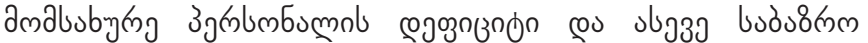

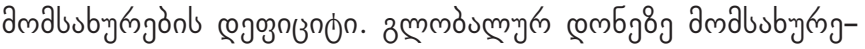

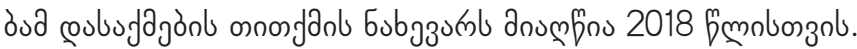

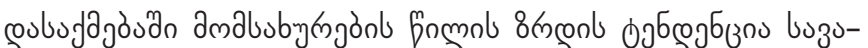

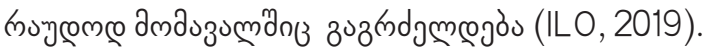

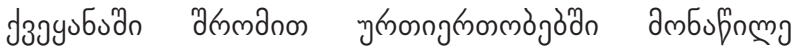

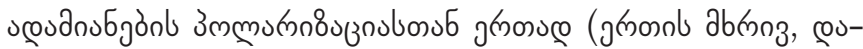

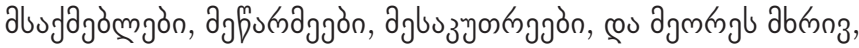

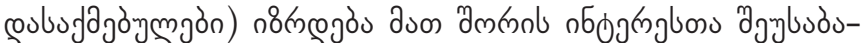

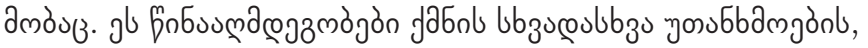

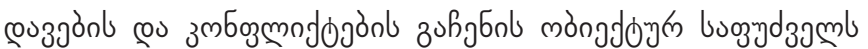

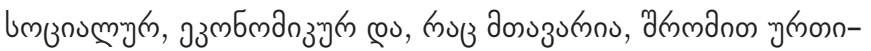

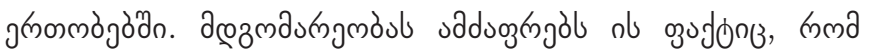

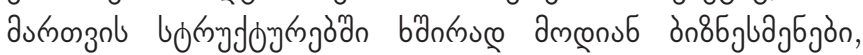

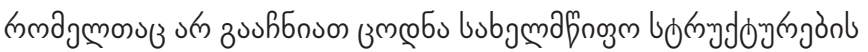

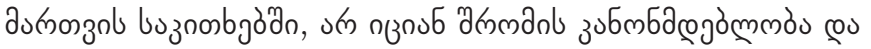

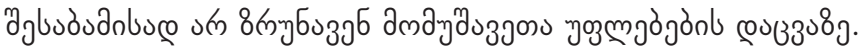

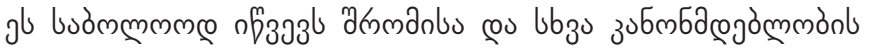

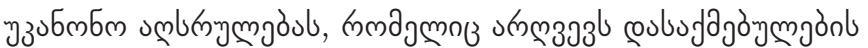

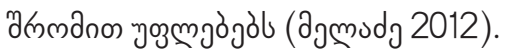

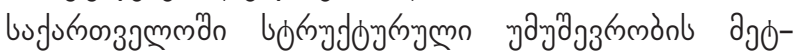

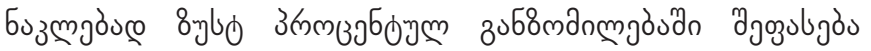

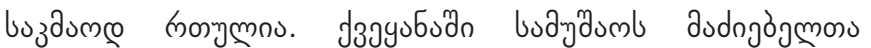

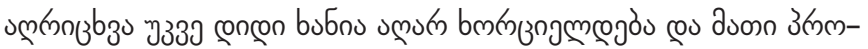

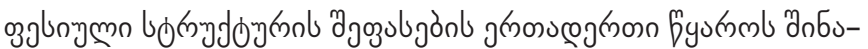

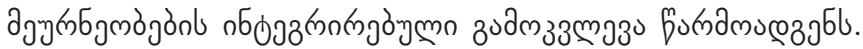

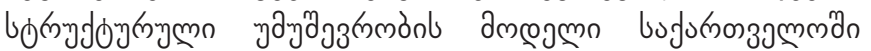

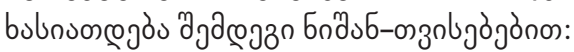

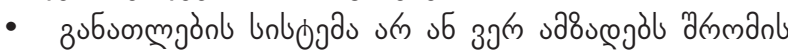

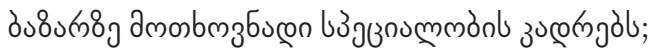

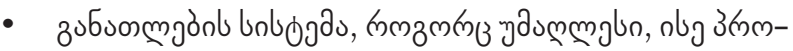

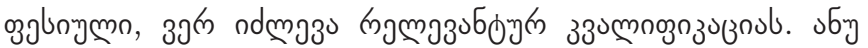

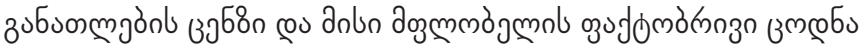

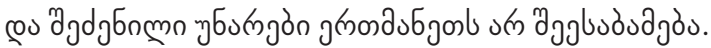

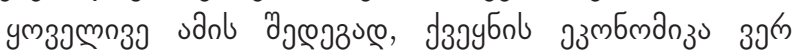

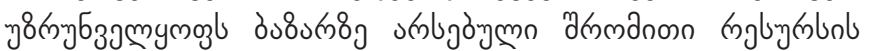

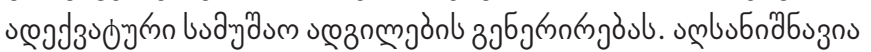

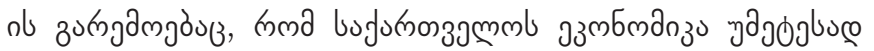

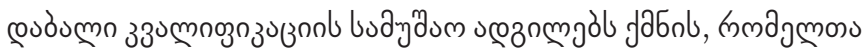

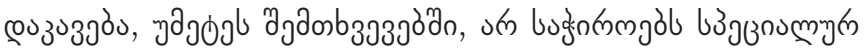
3ubsongmojol.

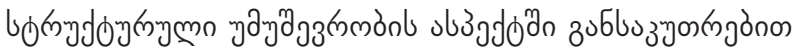

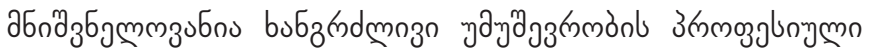

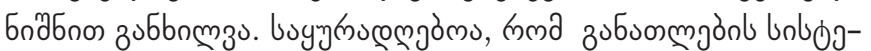

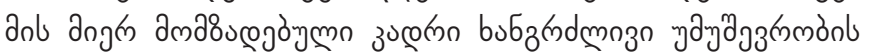

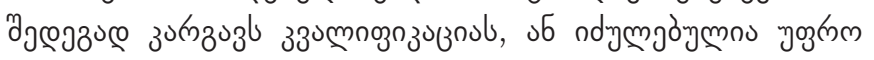

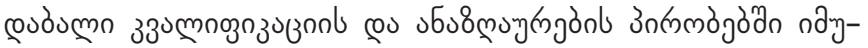

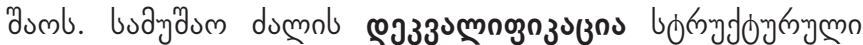

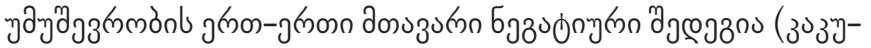
mos, 3o3ubodg 2016, 33. 31).

\section{юозวณงวง №1}

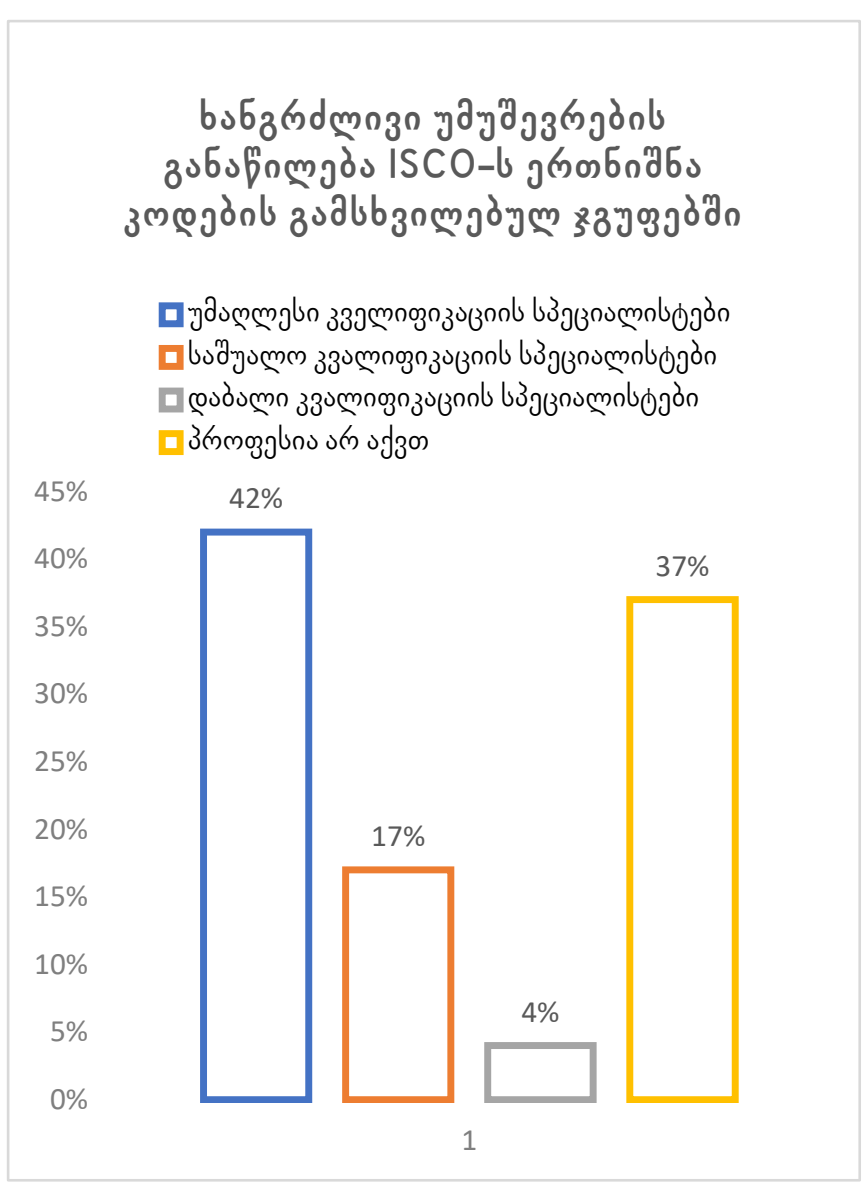

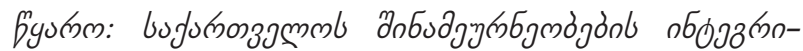

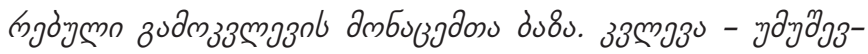

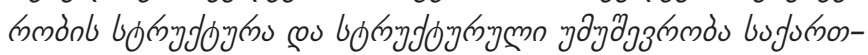

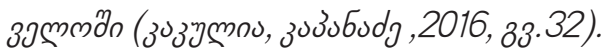

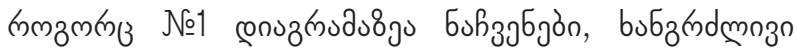

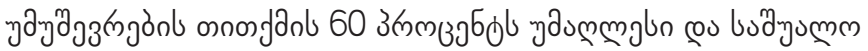

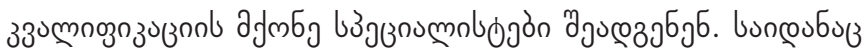

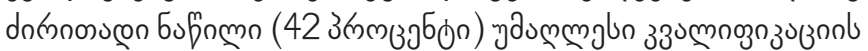

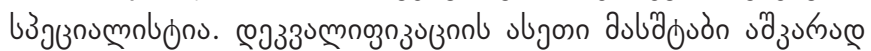

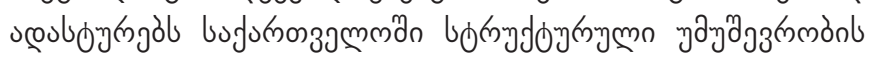

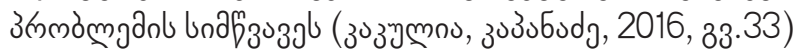

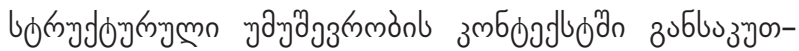

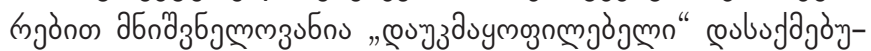

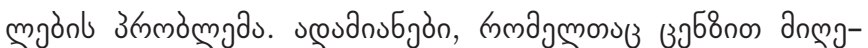
бचm

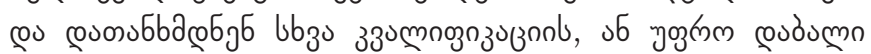

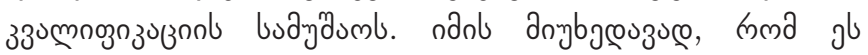

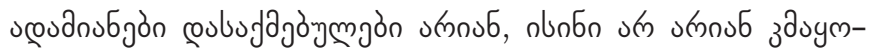

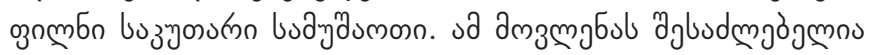

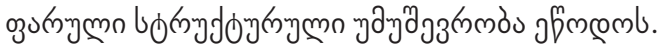

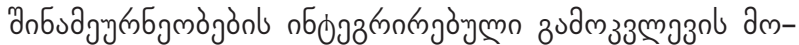

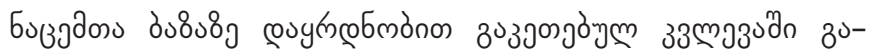

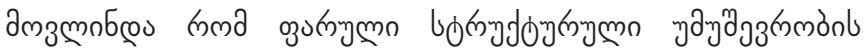




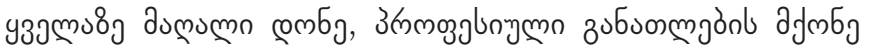

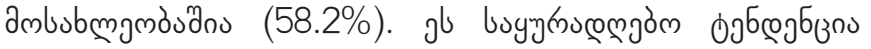

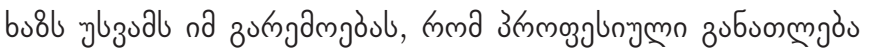

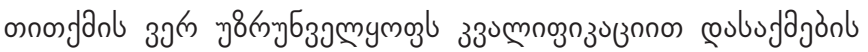

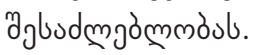

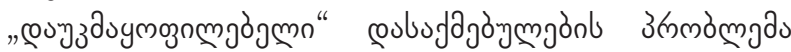

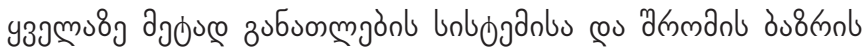

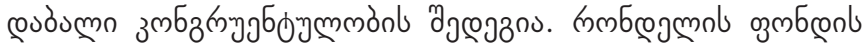

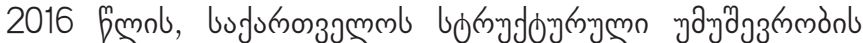

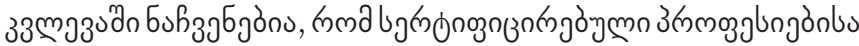

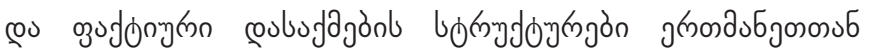

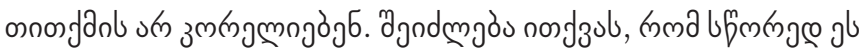

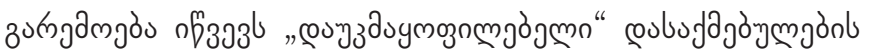

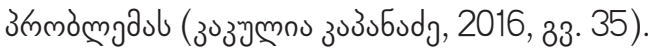

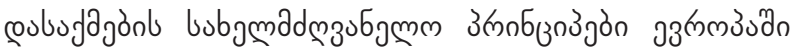

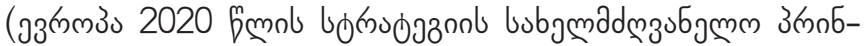

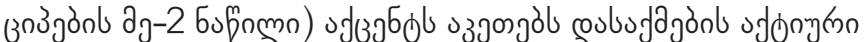

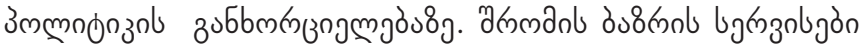

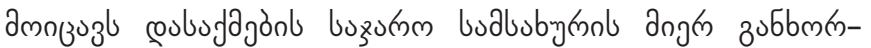

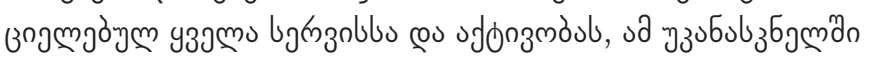

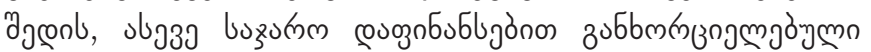

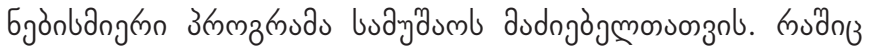

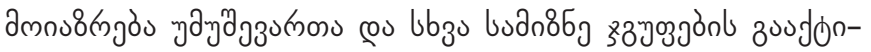

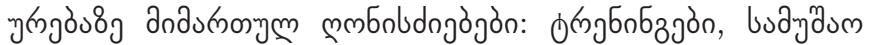

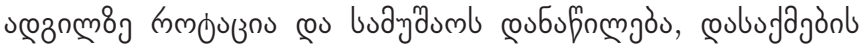

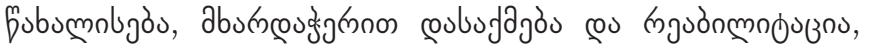

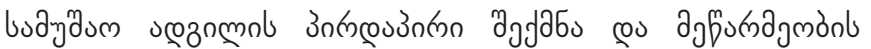

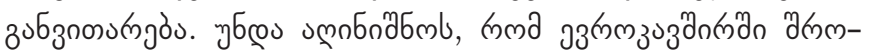

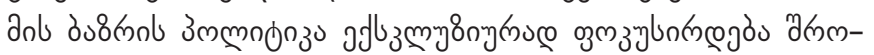

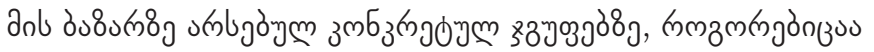

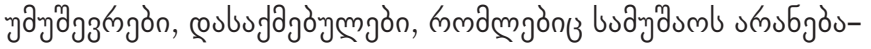

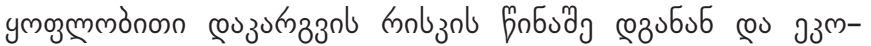

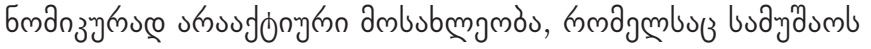
coufygas bynt.

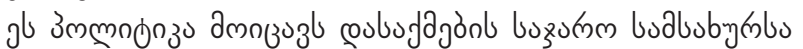

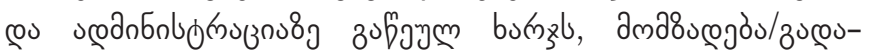

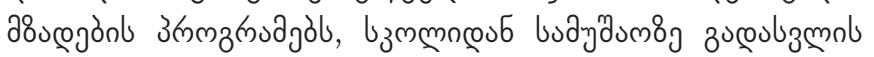

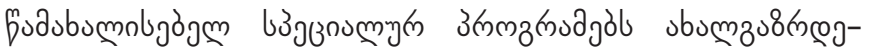

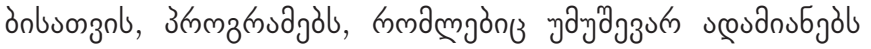

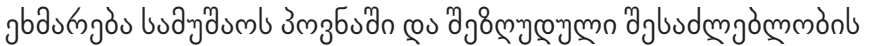
a

2016 fmol 4 s3

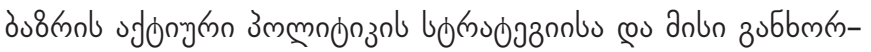

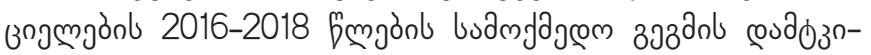

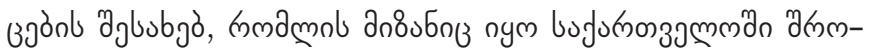

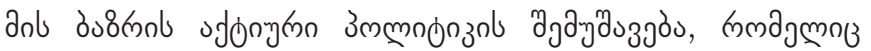

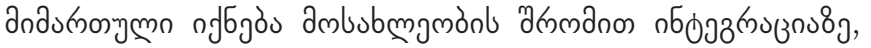

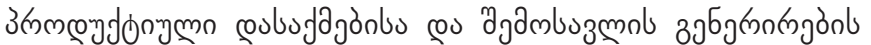

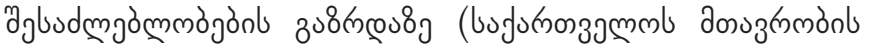

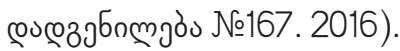

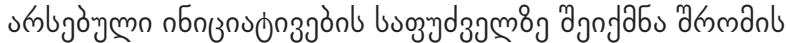

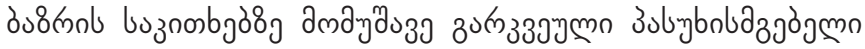

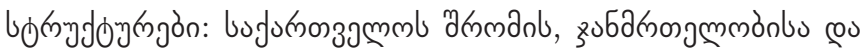

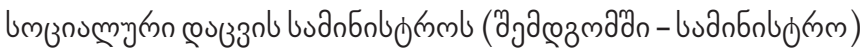

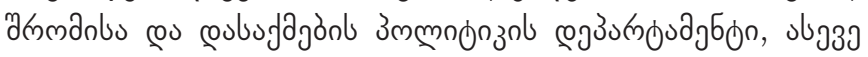

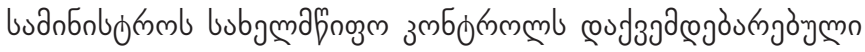

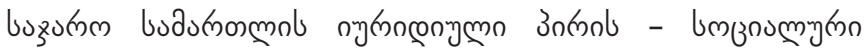

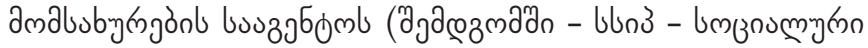

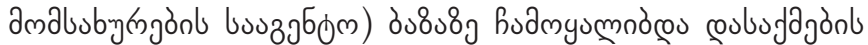

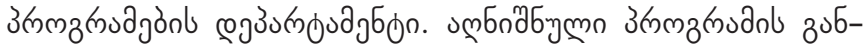

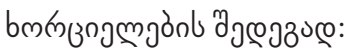

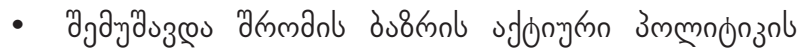

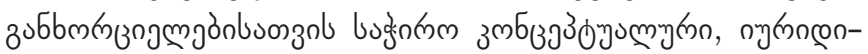

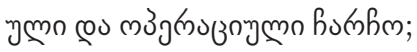

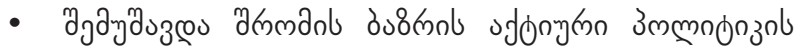

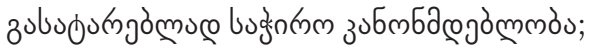

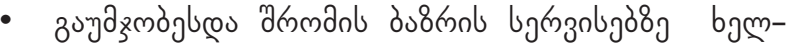

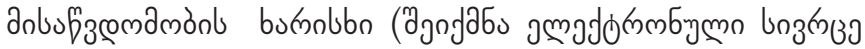

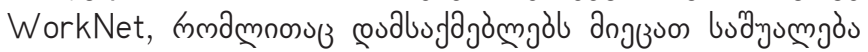

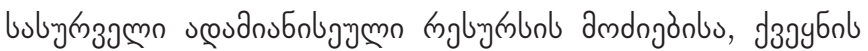

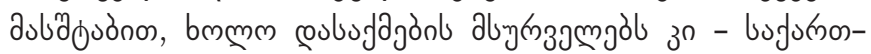
заलmm

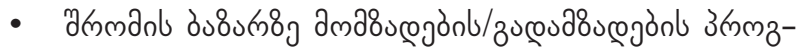

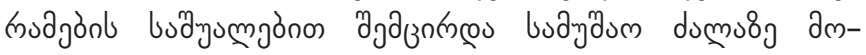

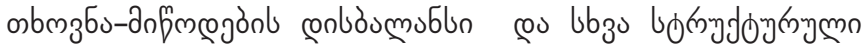

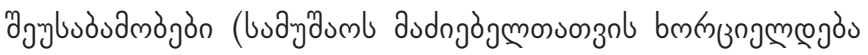

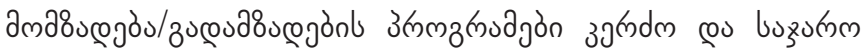

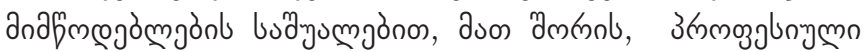

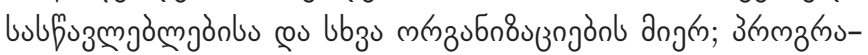

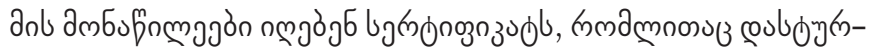

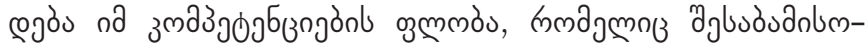

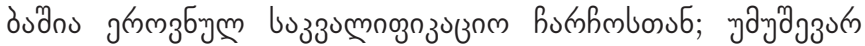

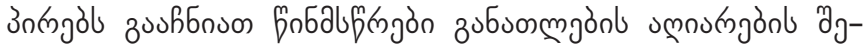

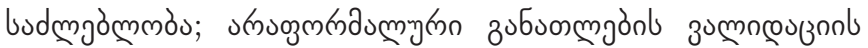

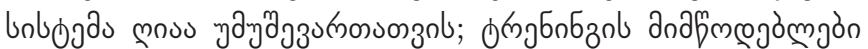

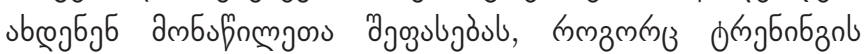
coubufynolan, n o

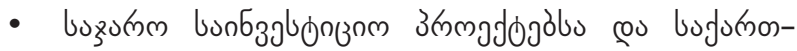

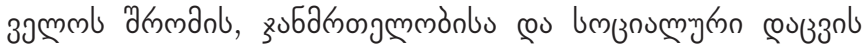

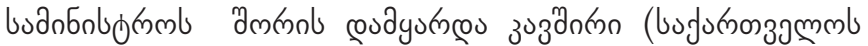

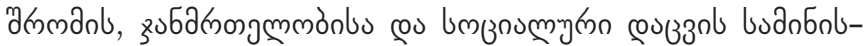

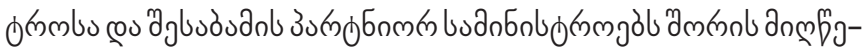

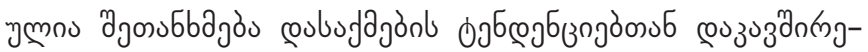

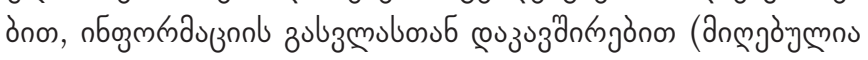

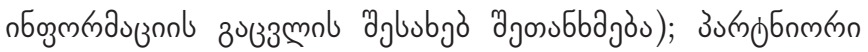

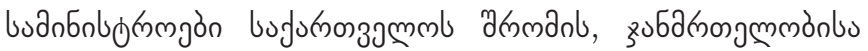

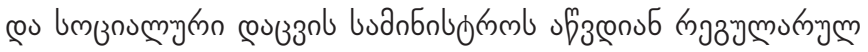

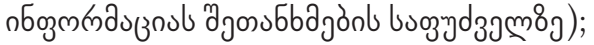

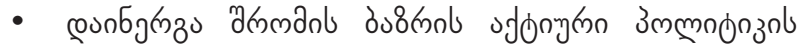

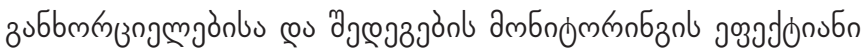




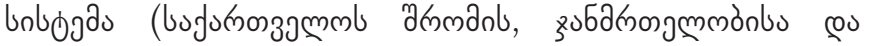

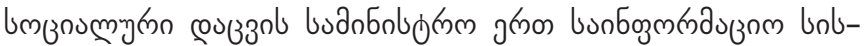

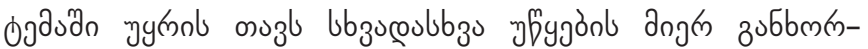

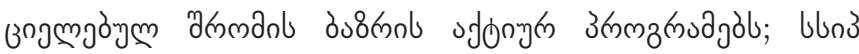

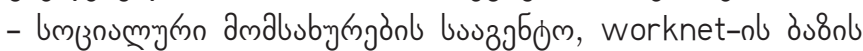

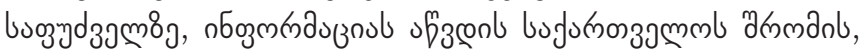

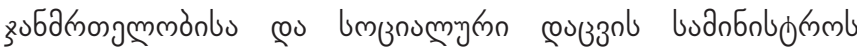

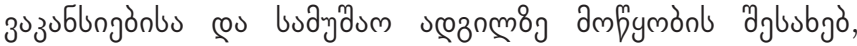

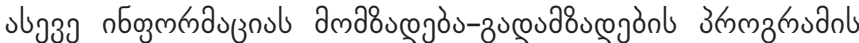

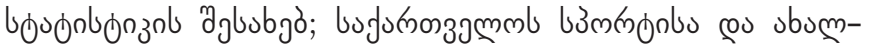

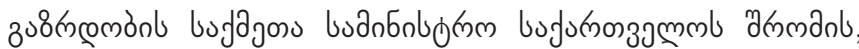

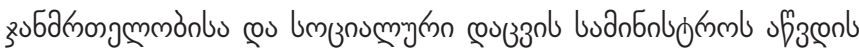

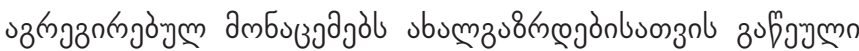

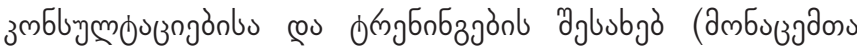

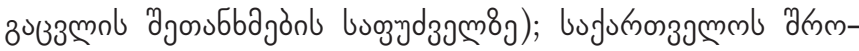

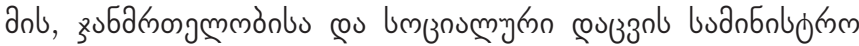

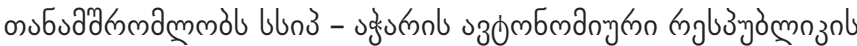

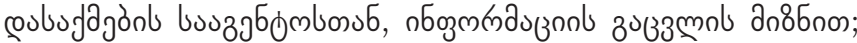

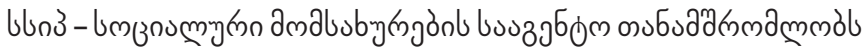

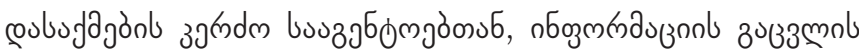

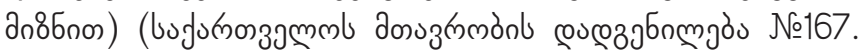
2016).

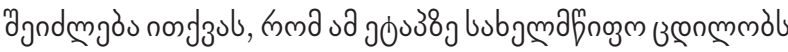

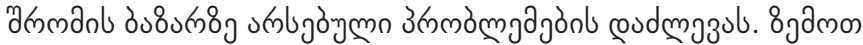

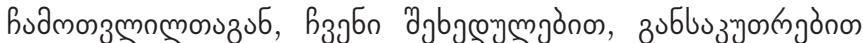

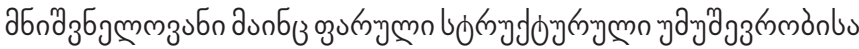

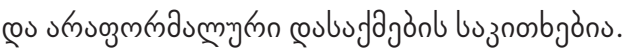

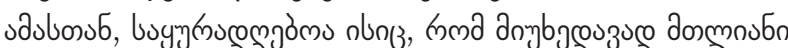

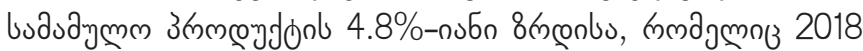

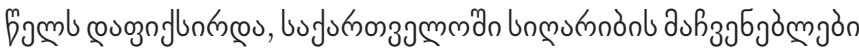

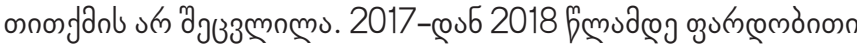

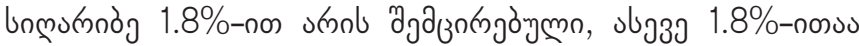

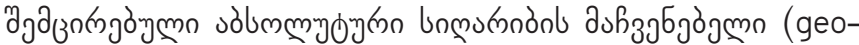

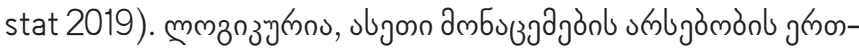

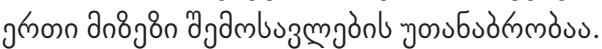

umzви

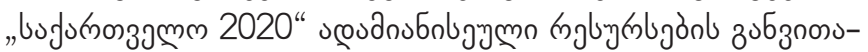

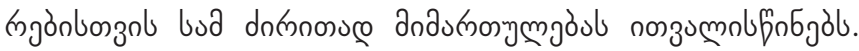

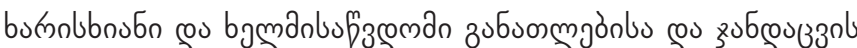

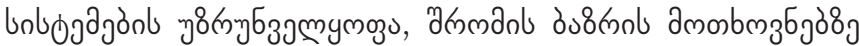

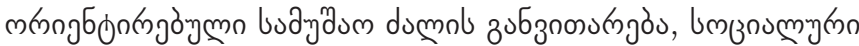

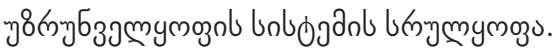

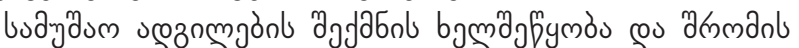

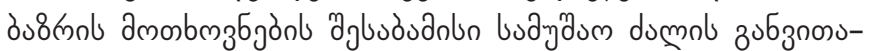

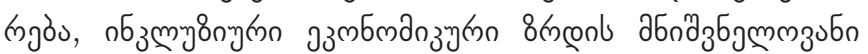

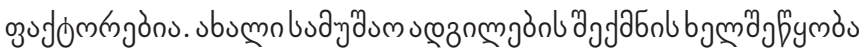

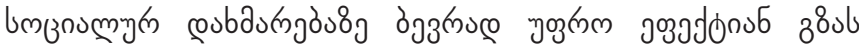

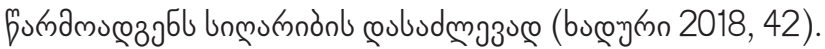

\section{esu335s}

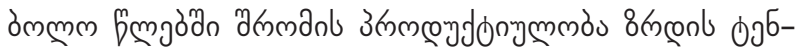

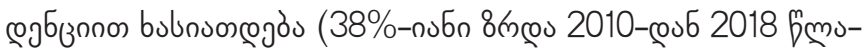

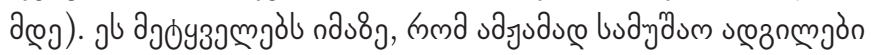

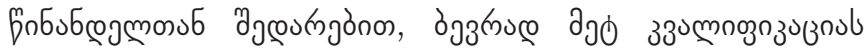

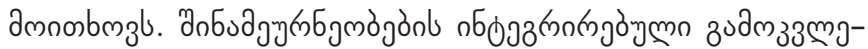

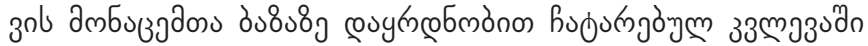

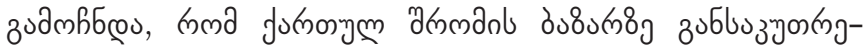

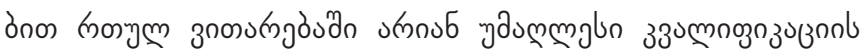

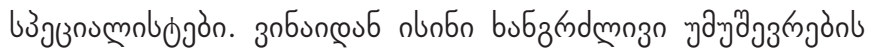

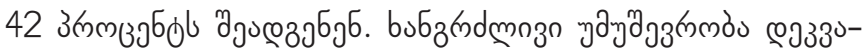
mnoznzuznol zuam

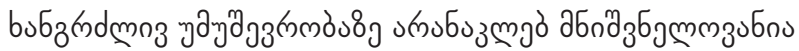

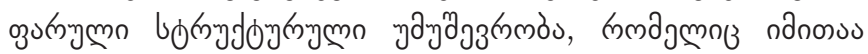

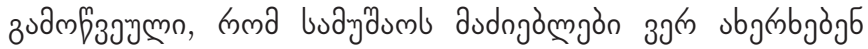

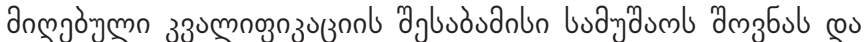

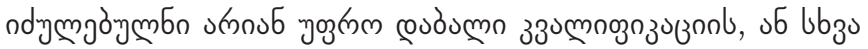

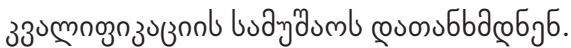

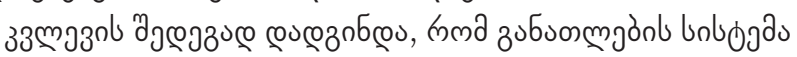

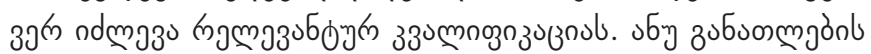

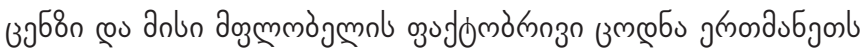

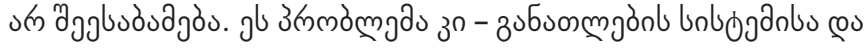

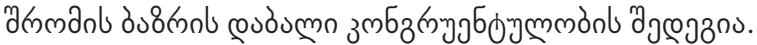

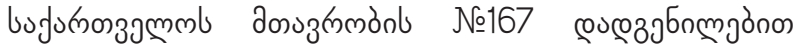

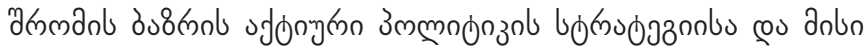

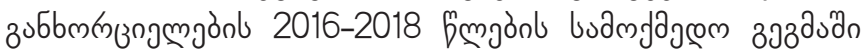

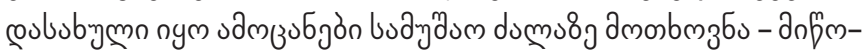

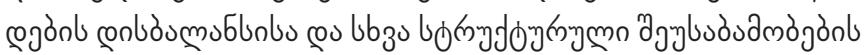
gjloazong gàmuce.

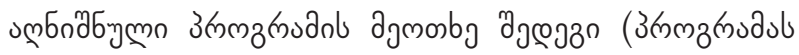

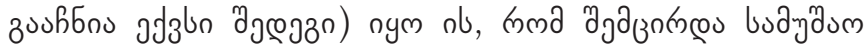

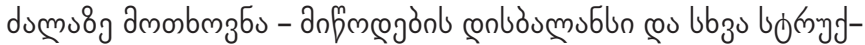

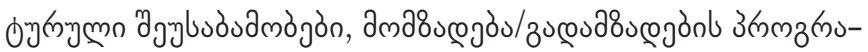
agònb buàyumgànon.

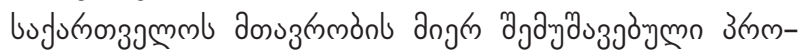

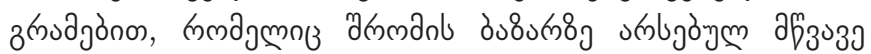

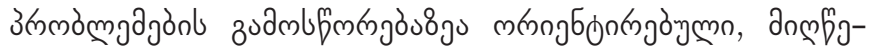

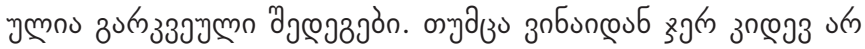

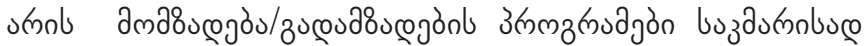

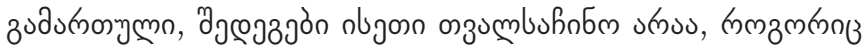

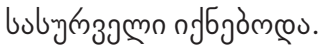

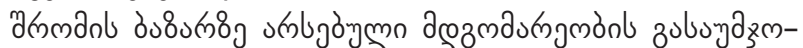

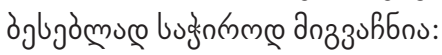

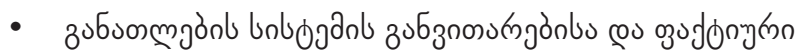

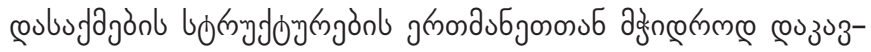
gntrgò; 


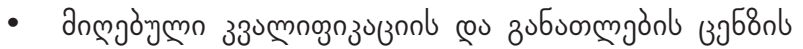

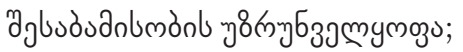

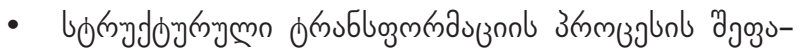

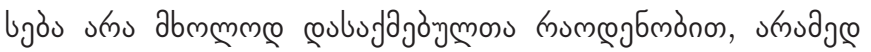

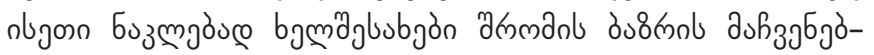

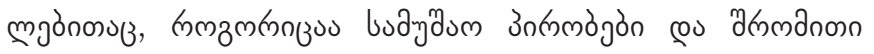

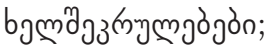

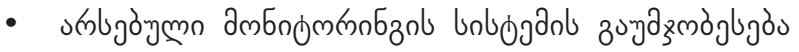

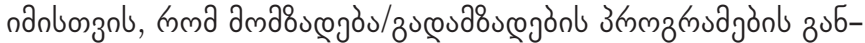

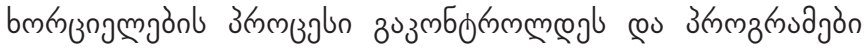

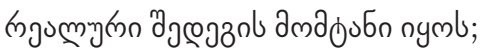

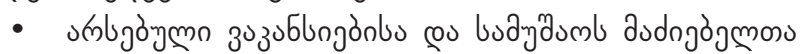

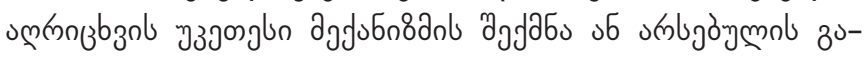

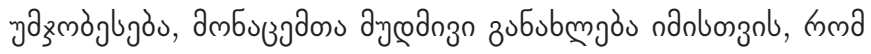

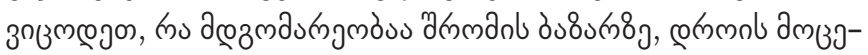

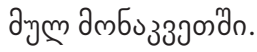

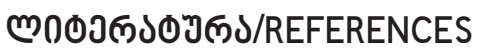

Aliyev H. (2015). Post-Soviet informality: towards theory-building. Enlighten - Research publications by members of the University of Glasgow p.8. 06.11.2019 http://eprints.gla.ac.uk/153383/1/153383.pdf

Arnania-Kepuladze T. (2015). Labor Market Formal Institutions: Structure and Specification. [Shromis Bazris Formaluri Institutebi: Struqtura da Makhasiateblebi]. Akaki Tsereteli State University. 1st International Conference (November 1314, 2015) «Economic, Legal and Social Problems of Modern Development» Kutaisi. 6 p. (In Georgian).

Geostat. (2019). Employment and Unemployment in Georgia. annual report of 2018. https://www.geostat.ge/media/23683/ Employment-and-Unemployment--2018-annual-\%28eng\%29.pdf

Geostat. (2019). Living conditions. https://www.geostat.ge/en/modules/categories/192/living-conditions

International Labour Organization (2018). COLLECTIVE AGREEMENTS: EXTENDING LABOUR PROTECTION, pp. 3-4, 26, https:// www.ilo.org/wcmsp5/groups/public/---dgreports/---dcomm/documents/publication/wcms_633672.pdf

International Labour Organization (2019). World Employment Social Outlook. https://www.ilo.org/wcmsp5/groups/public/--dgreports/---dcomm/---publ/documents/publication/wcms_670542.pdf

Kakulia M. Kapanadze N. (2016) Structure of Unemployment and Structural Unemployment in Georgia. published by FriedrichEbert-Stiftung (FES). pp. 31-37. https://gfsis.org/files/library/pdf/2456.pdf

Khaduri N. (2018) Factors of Inclusive Growth and Challenges faced by the Economy of Georgia [Inkluziuri zrdis faqtorebi da sakartvelos ekonomikis tsinashe arsebuli gamotsvevebi], Journal "Ekonomisti", Publishing House of Paata Gugushvili Institute of Economics of TSU, Vol. 14, No. 1, pp. 37-45. (In Georgian).

Labor force participation rate, total (\% of total population ages 15+) (modeled ILO estimate) 06.11.2019 https://data. worldbank.org/indicator/SL.TLF.CACT.ZS

Makalatia G. (2014). Statistical Survey of Standard of Living of Georgian Population [Sakartvelos mosakhleobis ckhovrebis donis statistikuri kvleva]. p.105. (In Georgian).

Meladze E. (2012). Regulation of Labor Relations and Development of Social Dialogue in Georgia [shromiti urtiertobebis regulireba da socialuri dialogis ganvitareba sakartveloshi]. Pp. 15-23. (In Georgian).

Namchavadze B. (2019) Prospects for Overcoming the Problem of Unemployment, Journal Forbes Georgia, 28.12.2019, https://forbes.ge/news/7287/Prospects-for-Overcoming-the-Problem-of-Unemployment

Resolution of the Government of Georgia N167 (2016) on Approval of Strategy on Active Labour Market Policy and its Action Plan for 2016-2018. (In Georgian). https://www.matsne.gov.ge/ka/document/download/2816365/0/ge/pdf

Smith and Stenning. (2006). Beyond household economies: articulations and spaces of economic practice in postsocialism, Progress in Human Georgraphy 30, p.192 